\title{
Winter sports special issue
}

\author{
Tom Allen ${ }^{1}$ (I) $\cdot$ Øyvind Sandbakk ${ }^{2} \cdot$ Stefan Josef Lindinger $^{3}$
}

Published online: 26 October 2017

(C) International Sports Engineering Association 2017

In all winter sports, equipment is essential for athlete performance and safety. Research into Sports Engineering, therefore, has a direct and tangible impact on the performance and safety of winter sports athletes. Prior to Sochi 2014, Sports Engineering published its first special issue on winter sports in Volume 16 Issue 4. Building on this success, Sports Engineering have dedicated this issue to winter sports, prior to PyeongChang 2018.

An increasing number of Sports Engineering studies are focussing on winter sports. Our call for papers to this special issue resulted in seventeen submissions. Nine of these submissions are included in this issue and some of those remaining will be distributed throughout future issues. In this editorial, we provide a brief overview of these nine papers, and outline how they have contributed to the scope of this special issue.

Many of the papers within the issue bridge the gap between the analysis of equipment and the behavior of the athlete. Indeed, they relate to the development of new

Tom Allen

t.allen@mmu.ac.uk

Øyvind Sandbakk

Oyvind.sandbakk@ntnu.no

Stefan Josef Lindinger

stefan.lindinger@sbg.ac.at

1 Manchester Metropolitan University, Chester Street, Manchester M1 5GD, UK

2 Department of Neuromedicine and Movement Science, Centre for Elite Sports Research, Norwegian University of Science and Technology, Smistadvegen 11, 7026 Trondheim, Norway

3 Department of Food and Nutrition and Sport Science, University of Gothenburg, Läroverksgatan 5, PO Box 300, SE405 30 Gothenburg, Sweden types, or modifications, of equipment, as well as adaptations of movement patterns and new testing procedures in different winter sports. All but two papers involve human participants, indicating the importance of the end-user in winter Sports Engineering. Overall, two main sporting themes are covered: ice hockey and snow sports, including alpine skiing, cross-country skiing and freestyle snowboarding. Areas of study include performance, comfort and safety, with methods covering elements of materials, finite element analysis, biomechanics, physiology, modelling, sensors and instrumentation.

The first three papers are focused on ice hockey and involve motion capture analyses of players. Kays and Smith investigated the effect of stick stiffness on puck speed for slap and wrist shots, combining player testing with finite element modelling. For slap shots, puck speed was inversely proportional to stick stiffness. They found that a more compliant stick bent more as the player loads it against the ice, storing more strain energy in the shaft which is then transferred to the puck as kinetic energy. The opposite trend was found for wrist shots, with puck speed increasing with stick stiffness as less energy was transferred to shaft deflection at impact. Renaud and colleagues compared the starts of high- and low-calibre skaters, using motion capture on an ice rink. Despite similar strength profiles between the two groups, more experienced, highcalibre skaters were able to achieve greater forward velocity in their starts. This was due to these skaters achieving a greater vertical centre of mass acceleration, allowing them to achieve greater traction on the ice and higher stride rates. Frayne and Dickey investigated the influence of pad design on goaltender leg kinematics during butterfly manoeuvres. The speed goalkeepers were able to perform their butterfly drops was dependent on pad design; findings having implications for the development 
and choice of pad design, from both a safety and performance perspective.

The remaining six papers are concerned with snow sport. Colonna et al. investigated how thermo-formation of ski boots can improve comfort. Stiff plastic boots are required for skiing, to protect the foot and control the ski, although these do not always provide a comfortable fit. An emerging practice is that of thermo-formation-heat moulding the boot for customisation. Colonna et al. found that boot shell material, heating time and cooling procedures all affected boot deformation, and hence pressure on the foot. Safety is another important issue in snow sports, particularly freestyle skiing and snowboarding, where a fall from a jump can result in serious injury. Petrone and coworkers advocate a jump engineered to have a constant equivalent fall height, no matter where the user lands. They modelled one of these jumps, built it on a ski slope and tested it using accelerometers mounted on a snowboarder's back and board. Experimental results agreed with model predictions, providing evidence to support their suggested approach to designing, and building, safer jumps.

Two papers were concerned with ski-snow friction, which has implication for performance and safety. Matveev used an analytical model to predict flat-ski friction in steady horizontal gliding, showcasing the influence of different parameters, such as velocity and snow temperature. Budde and Himes adopted an experimental approach, using a bespoke tribometer to measure friction between cross-country ski bases and snow, while quantifying the effect of wax and base texturing treatments.

Seeberg and co-workers developed, and showcased the feasibility, of a multi-sensor system for analysing crosscountry skiing techniques, consisting of a heart rate sensor, Global Navigation Satellite Systems data and seven Inertial Measurement Units placed at multiple locations on the body. Employment of hard decision rules based on the correlation between arms and legs could detect sub-techniques in classical cross-country skiing with high sensitivity and precision, indicating that such systems could be useful tools for athletes and coaches, as well as for testing of equipment. In the final paper, Ørtenblad and Jensen employed a special double poling ergometer to test predictors for 6-min all-out performance in cross-country skiers. They highlighted that these skiers should focus on improving their technique to increase gross mechanical efficiency rather than maximal oxygen uptake.

We would like to thank all authors for their contributions to this special issue that will be published before PyeongChang 2018. We hope that this special issue will stimulate further research and development on winter sports equipment, and we welcome a greater number of submissions on the topic of winter sports to Sport Engineering in the future. 